as the energy spectrum of turbulence governed by Kolmogorov's $k^{-5 / 3}$ law was concerned.

But Kolmogorov's theory contained the seeds of its own destruction, as was apparently recognized by Landau in discussions following the presentation of Kolmogorov's papers to the Russian Academy. The local rate of dissipation of energy per unit volume $\bar{\varepsilon}$ is itself subject to spatial fluctuations, and, in subregions of the flow where $\bar{\varepsilon}$ is much greater than its spatial average, the local statistics should reflect this local value rather than the mean value $\bar{\varepsilon}$. Indeed, in regions where $\bar{\varepsilon}$ is large, the process of cascade of energy from large to small scales is more intense, smaller scales of motion are generated, and the rate of viscous dissipation becomes even larger. This leads to the problem of intermittency of turbulent dissipation, a problem that was recognized by Kolmogorov himself in 1962 (Journal of Fluid Mechanics 13, 82-85) and has since attracted increasing attention.

All of this is discussed by Frisch in a stimulating and imaginative manner. $\mathrm{He}$ begins with a qualitative discussion of symmetry-breaking instabilities of a typical flow as the Reynolds number (Re) is increased from small values, and argues that in the asymptotic turbulent regime of very large $\mathrm{Re}$, although all symmetries are broken in detail, a new statistical symmetry (essentially homogeneity and isotropy) emerges in relation to the small scales of motion. He then presents a novel discussion of the vital process of energy transfer (or 'cascade') from large to small scales. This leads to a discussion of the seeds of chaos in simple dynamical systems, and to a sophisticated introduction to some of the probabilistic techniques used in the theory of turbulence.

In the heart of the book, Frisch presents and develops his own reinterpretation of Kolmogorov's theory and contemporary theories incorporating the effects of intermittency. He bases his presentation on two empirical laws, the famous 'two-thirds law' relating to mean-square of the velocity difference between two points in the flow, and the 'law of finite energy dissipation', which states that $\bar{\varepsilon}$ tends to a constant independent of $\nu$ as $\nu$ tends to 0 . The first law is well supported by experiments, the second less so; if this second law is not valid - a possibility that Frisch does not care to contemplate - the whole Kolmogorov construction is a house built on sand.

One of the most illuminating discussions of intermittency is that involving the ' $\beta$ model' introduced by Frisch, Sulem and Nelkin in 1978. As the energy cascades, the volume in which 'active eddies' are present is supposed to reduce each step of the cascade by a factor $\beta<1$; this leads to a fractal distribution for $\varepsilon(x)$ from which higherorder statistics can also be deduced. Unfortunately, the theory is not in accord with subsequent experiments; to achieve agree- ment, as Frisch shows, it is necessary to adopt a 'multifractal' rather than a simple fractal viewpoint, involving a continuum of coexisting fractal dimensions. At this point, many more earth bound researchers will part company with Frisch (and other multifractalists), unprepared to accept that the ultimate nature of turbulence may hinge on such an alien concept.

Much more promising, I believe, is the more conventional and physically appealing picture of turbulence as a random superposition of stretched vortex filaments, the 'sinews of turbulence'. Direct numerical simulations have been pointing in this direction for some years, and experiments by Couder and others (Douady, Couder and Brachet, Physical Review Letters 67, 983; 1991) lend credence to this view. The wheel of turbu-

\section{Holes unearthed}

\section{Stephen Battersby}

Gravity's Fatal Attraction: Black Holes in the Universe. By Mitchell Begelmen and Martin Rees. W. H. Freeman: 1996. Pp. 246. £19.95, \$32.95, £19.95.

"It's black, and it looks like a hole. I'd say it's a black hole."

SIDNEY Harris's white-coated cartoon astronomer makes this deadpan satire on scientific coinage and deduction while showing her colleague a picture of a star field with a big round gap in it. Although we do not yet have any images that are so

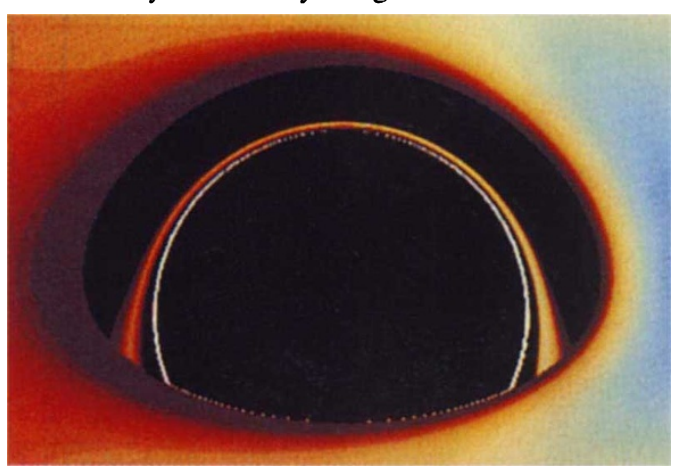

Simulated close-up view of a black hole and its disk of accreting gas, relativistically distorted by gravity and the high orbital speeds.

unequivocal, most astronomers assume that black holes probably do exist, either on the weight of circumstantial evidence or because their colleagues tell them so. But since 1963, when Roy Kerr calculated the properties of spinning black holes, we have been in the odd position of knowing exactly what a whole class of objects is like without knowing whether a single one exists.

When will the evidence accumulate to a point beyond reasonable doubt? It already has, according to Begelman and Rees. lence is coming full circle as we witness a return, albeit at a highly sharpened level, to a consideration of concepts (vortices and vortex interaction) with which Kelvin would have felt at ease.

Frisch concludes his monograph with a long chapter providing a guided tour of further reading in the subject, an extremely useful aid for students whose appetites may have been whetted by this personal, highly unconventional and provocative account. This book will be a source of inspiration for theoretically inclined researchers, but will provide little comfort for practising engineers.

H. K. Moffatt is in the Department of Applied Mathematics and Theoretical Physics, University of Cambridge, Silver Street, Cambridge CB3 9EW, UK.

Exemplifying the book's confident stance, they say: "But we now know that black holes are not mere theoretical constructs; they exist in profusion and account for many of the most spectacular astronomical discoveries of recent times".

This certainty is prompted by a discovery that was made shortly before the book went to press. In the nucleus of the galaxy NGC4258, there are gas clouds in which water vapour forms natural masers. They trace out a warped disk of material orbiting at enormous velocities, betraying such a dense central concentration of mass that "the only plausible alternative is a single massive black hole". Unfortunately, the figures that illustrate this are by far the most confusing in the book; perhaps the news was so hot that they had to be printed at high speed. A few minutes of sweaty decipherment smudged the ink on two of them, strengthening my suspicion.

Other than this, there is little to criticize here and plenty to delight in, from the history (apparently one Revd John Michell was the first to postulate black holes in 1784 , beating Laplace by ten years) to the well illustrated oddities of general relativity. Rigorous and thorough but not dry, the book describes holes of all shapes and sizes - all sizes, anyway from the still-improbable primordial subatomic holes, shining by Hawking radiation, to the billion-solar-mass holes that power quasars.

Black holes have lost part of their traditional mystique - the question of their existence - but I am sure there is plenty left to keep them popular.

Stephen Battersby is an assistant editor at Nature. 